\title{
Near-Infrared AIE Dots with Chemiluminescence for Deep-Tissue Imaging
}

\author{
Chenchen Liu, ${ }^{\dagger} \S$ Xiuxia Wang, ${ }^{\ddagger}{ }^{\S}$ Junkai Liu, ${ }^{\dagger}$ Qiang Yue, ${ }^{\ddagger}$ Jacky W. Y. Lam, ${ }^{\dagger}$ Liang Luo, ${ }^{*}{ }^{\ddagger}$ and Ben \\ Zhong Tang ${ }^{*} \dagger, \|, \perp$
}

'Department of Chemistry, Hong Kong Branch of Chinese National Engineering. Research Center for Tissue Restoration and Reconstruction, Institute for Advanced Study, Department of Chemical and Biomedical Engineering, Division of Life Science and State Key Laboratory of Molecular Neuroscience, The Hong Kong University of Science and Technology, Clear Water Bay, Kowloon, Hong Kong, China

${ }^{\ddagger}$ National Engineering Research Center for Nanomedicine, College of Life Science and Technology, Huazhong University of Science and Technology, Wuhan 430074, China

'Center for Aggregation-Induced Emission, State Key Laboratory of Luminescent Materials and Devices, SCUT-HKUST Joint Research Institute, South China University of Technology, Guangzhou 510640, China

${ }^{\perp}$ Ming Wai Lau Centre for Reparative Medicine, Karolinska Institute, Hong Kong, China

\section{Supporting Information}

\begin{abstract}
Near-infrared (NIR) chemiluminescence (CL) emission is highly favourable for deep-tissue imaging, but chemically conjugated NIR CL emitters with aggregation-induced emission (AIE) property for biotechnology are rarely reported. Herein, we designed an AIE-active NIR CL emitter TBL by conjugating luminol unit with electron-accepting benzothiadiazole and electron-donating triphenylamine, and subsequently prepared TBL dots using F127 as the surfactant. The CL emission of TBL dots can last continuously for over 60 minutes, and can be employed for quantitative (in vitro) and qualitative (in vivo) detection of ${ }^{1} \mathrm{O}_{2}$ Strikingly, the NIR CL emission can penetrate through tissues with a total thickness of over $3 \mathrm{~cm}$, exhibiting significantly better performance than NIR fluorescence emission and blue CL emission. Moreover, the successful differentiation of tumor and normal tissues by TBL-based CL imaging in vivo also paves the way for CL-guided cancer diagnosis and surgery.
\end{abstract}

Fluorescence (FL) is widely used in real-time visualization of biomedical processes with great sensitivity and high resolution. ${ }^{1-3}$ However, the penetration depth of FL is rather limited due to the requirement of excitation light and the autofluorescence from the biological structures. ${ }^{4}$ Although red and near-infrared (NIR) FL materials are popularly developed to reduce the tissue autofluorescence, ${ }^{5}$ to achieve high penetration depth still remains a great challenge, especially considering the inadequate penetration of excitation light. In contrast, chemiluminescence (CL) usually has a higher penetration depth than FL, because no excitation light is required and the background autofluorescence can be avoided, therefore holding great promise for deep-tissue imaging. ${ }^{6-7}$

The energy required to generate visible or NIR CL emission $(400-1000 \mathrm{~nm})$ is around $30-70 \mathrm{kcal} \mathrm{mol}^{-1}, 8$ while the opening reaction of peroxide groups (-O-O-) can release energy of around $60 \mathrm{kcal} \mathrm{mol}^{-1}$, so that peroxide groups are frequently utilized in CL reactions. ${ }^{9-10}$ For example, when the well-known CL emitter luminol is oxidized by reactive oxygen species (ROS), blue light can be observed during the decomposition of unstable peroxide intermediates. ${ }^{11}$ However, blue light is typically limited in tissue penetration. It is crucial to transfer the blue emission of luminol into the NIR region for enhanced penetration depth and efficiency, through deliberate molecular design principles.

The CL emission originates from the excited reaction product or the excited fluorescent acceptor through energy transfer. If $\mathrm{CL}$ comes from the fluorescent product, the CL quantum yield $\left(\Phi_{\mathrm{CL}}\right)$ is expressed as: $\Phi_{\mathrm{CL}}=\Phi_{\mathrm{R}} \times \Phi_{\mathrm{ES}} \times \Phi_{\mathrm{F}},{ }^{8}$ where $\Phi_{\mathrm{R}}$ is the reaction yield, $\Phi_{\mathrm{ES}}$ reflects the ratio of the product accessing the excited state, and $\Phi_{\mathrm{F}}$ is the fluorescent quantum yield of the reaction product. The CL-generating reaction of luminol involves many electron-rich intermediates like anions and radicals, so the reaction yield can be enhanced by conjugating electron-withdrawing groups. Benzothiadiazole used herein is a strong electron acceptor that has been widely utilized in organic solar cells ${ }^{12}$ and organic light emitting diodes, ${ }^{13-14}$ and it can efficiently facilitate CL generation after being conjugated with luminol. In addition, many hydrophobic organic dyes suffer from aggregation-caused quenching (ACQ) effect due to strong $\pi-\pi$ stacking, and the emission could be largely quenched in aqueous solutions. For example, the solubility and fluorescent quantum yield of the Nile red-based luminol cassette are relatively low in water, which restricts its further application in biological systems. ${ }^{15}$ In contrast, fluorophores with aggregation-induced emission (AIE) properties exhibit intense emission in the aggregation state due to the restriction of their molecular motions, making them more favorable for bioimaging. ${ }^{16-17}$ Moreover, the addition of the electron-donating group triphenylamine can form a donor-acceptor structure, and enable the bathochromic shift of the emission of luminol-containing fluorophore. Indeed, the fluorescent quantum yield of triphenylamine-combined benzothiadiazole (TB) in aggregation state is very high because of its AIE property. ${ }^{18-19}$ According to these principles, a NIR CL emitter TBL was synthesized by conjugating TB with luminol (Figure 1A and Scheme S1), in which the emission could come from the whole reaction product directly or from luminol unit to the whole molecule through energy transfer. 
A
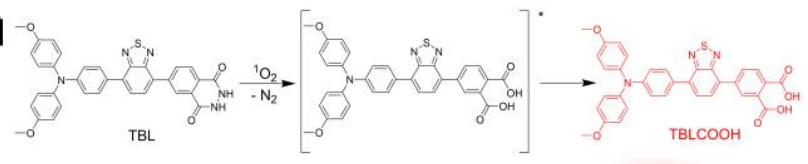

B
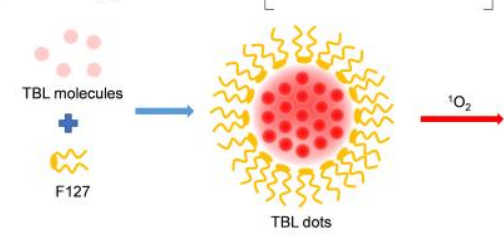

rescis

Figure 1. (A) The proposed CL generation mechanism of TBL oxidized by ${ }^{1} \mathrm{O}_{2}$. (B) Schematic illustration of the preparation of TBL dots and the generation of CL.

In addition, if the CL emission is generated from the acceptor fluorophore by energy transfer, the fluorescent quantum yield of the acceptor $\left(\Phi_{\mathrm{F}}\right)$ and the energy transfer efficiency $\left(\Phi_{\mathrm{ET}}\right)$ should be taken into consideration as well: $\Phi_{\mathrm{CL}}=\Phi_{\mathrm{R}} \times \Phi_{\mathrm{ES}} \times \Phi_{\mathrm{F}}{ }^{\prime} \times \Phi_{\mathrm{ET}}{ }^{8}$ The through-bond energy transfer was reported to be more efficient than the through-space energy transfer, ${ }^{15,20}$ which was also proved by the chemically conjugated AIE nanoparticle NTPE$\mathrm{PH} .{ }^{21}$ However, its blue CL emission is not ideal for deep-tissue imaging. Although some NIR CL systems have been developed by physically encapsulating the CL emitters with the NIR fluorescent materials in surfactant micelles, ${ }^{22-23}$ the preparation process is rather complicated and phase separation may occur during the long-term storage. More importantly, the energy transfer efficiency is much lower than the chemical conjugation system. Therefore, chemically conjugated TBL can not only facilitate the energy transfer, but also is favorable for deep-tissue imaging attributed to its NIR CL emission. In addition, TBL dots are effective in differentiating tumor and normal tissues, manifesting great potential for future CL-guided cancer diagnosis and therapy applications.
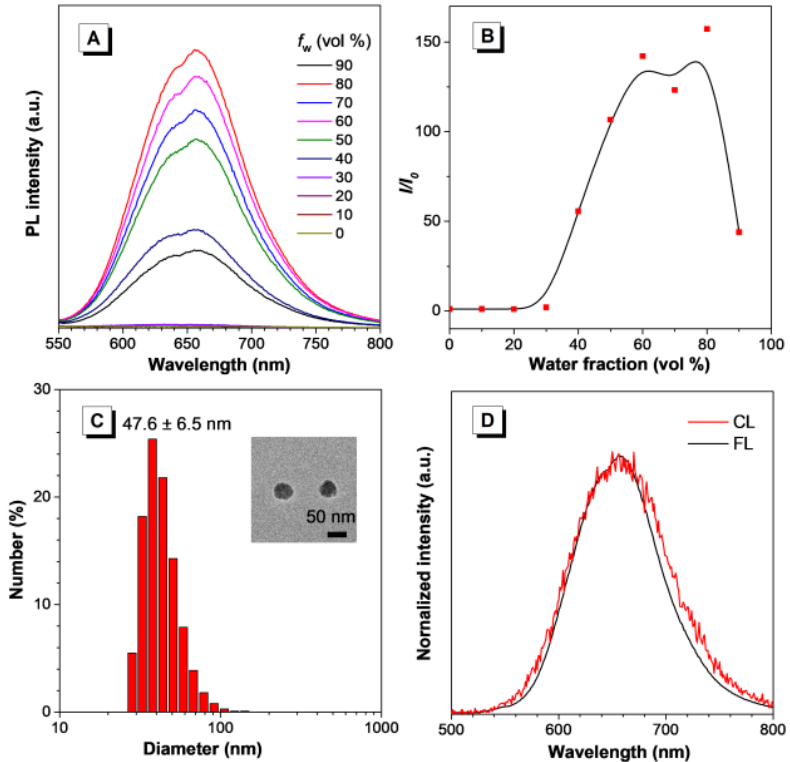

Figure 2. (A) PL spectra of TBL $\left(10^{-5} \mathrm{M}\right)$ in different $\mathrm{DMSO} / \mathrm{H}_{2} \mathrm{O}$ mixtures with the addition of water $\left(\lambda_{\mathrm{ex}}: 460 \mathrm{~nm}\right)$. (B) The plot of relative maximum emission intensity $\left(I / I_{0}\right)$ of TBL in different DMSO/ $\mathrm{H}_{2} \mathrm{O}$ mixtures. (C) Hydrodynamic size distribution of TBL dots in PBS solution ( $\mathrm{pH}=7.4)$. Inset: TEM image of TBL dots. (D) Normalized FL and CL spectra of TBL dots.

The AIE property of TBL was firstly evaluated in different DMSO/ $\mathrm{H}_{2} \mathrm{O}$ solutions. TBL was not emissive in DMSO solution because the energy was consumed by free molecular motion in the solution state. The emission increased gradually after more than $30 \%$ water was added, due to the restriction of molecular motion, ${ }^{16}$ indicating that TBL is AIE active. The fluorescent quantum yield of TBL in solid state was measured to be $12.5 \%$. (Figure 2A, $\mathrm{B})$. However, TBL could form large particles and precipitate in PBS solution ( $\mathrm{pH}=7.4)$, making them no longer suitable for further applications (Figure S11). To solve this problem, we utilized F127 as the surfactant to form TBL dots with diameters of around $50 \mathrm{~nm}$ (Figure 1B, 2C). It was well studied that singlet oxygen $\left({ }^{1} \mathrm{O}_{2}\right)$ could burn the luminol units to generate successive CL, ${ }^{21,24}$ so that we employed ${ }^{1} \mathrm{O}_{2}$ produced by mixing $\mathrm{H}_{2} \mathrm{O}_{2}$ and $\mathrm{NaClO}$ solutions ${ }^{25}$ to initiate the CL process of TBL dots. In principle, the luminol unit of TBL could form radicals in the reaction with ${ }^{1} \mathrm{O}_{2}$ first, then it was oxidized to form the unstable TBL peroxide. During the decomposition process, the excited TBLCOOH was populated directly or by the excited luminol unit through energy transfer. Subsequently, the NIR emission centered at $658 \mathrm{~nm}$ was generated when the excited TBLCOOH came back to the ground state (Figure 1, S13). Moreover, the CL spectrum of TBL dots matched well with their FL spectrum (Figure 2D), and the DFT calculation also demonstrated that the energy levels of TBL and its oxidation product were almost unchanged (Figure S14), indicating the CL emission might come from the excited TBLCOOH.
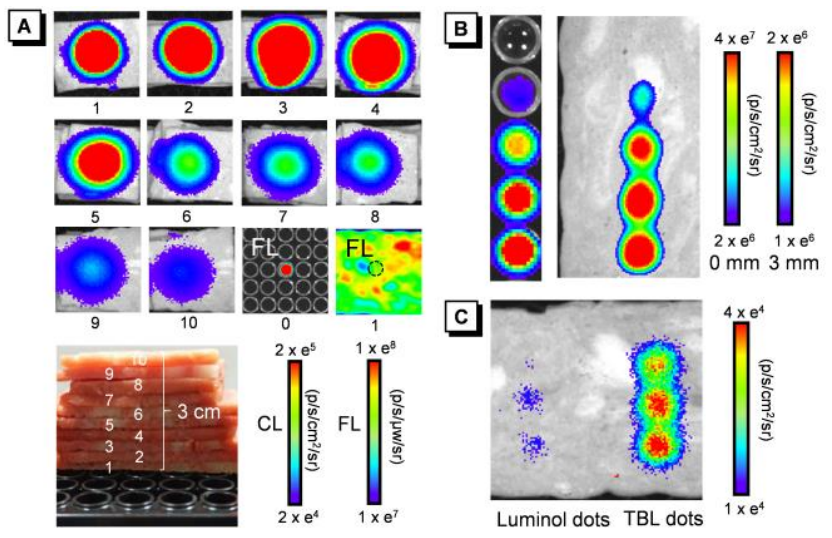

Figure 3. (A) $\mathrm{CL}$ images of TBL dots $(2 \mathrm{mM})$ with ${ }^{1} \mathrm{O}_{2}(150 \mathrm{mM})$ and FL images of TBL dots $(2 \mathrm{mM})$ covered by different slices of pork ham. The thickness of one slice pork ham is around $3 \mathrm{~mm}$. Binning: 8; Exposure time: $4 \mathrm{~min}$; f/Stop:1. (B) CL images of TBL dots $(2 \mathrm{mM})$ with different concentration of ${ }^{1} \mathrm{O}_{2}$ (left) and covered with one slice of $\sim 3.0 \mathrm{~mm}$-thick pork ham (right), the concentration of ${ }^{1} \mathrm{O}_{2}$ is $5 \times 10^{-4} \mathrm{M}, 1 \times 10^{-3} \mathrm{M}, 2.5 \times 10^{-3} \mathrm{M}, 5 \times$ $10^{-3} \mathrm{M}, 1 \times 10^{-2} \mathrm{M}$ from top to bottom. Binning: 4; Exposure time: $0.5 \mathrm{~min}$; f/Stop:1. (C) CL images of Luminol dots $(1.2 \mathrm{mM})$ and TBL dots $(1.2 \mathrm{mM})$ with ${ }^{1} \mathrm{O}_{2}(8 \mathrm{mM})$ covered by one slice of $\sim 3.0$ mm-thick pork ham. Binning:4; Exposure time: 4 min; f/Stop:1.

We next investigated the quantitative detection of ${ }^{1} \mathrm{O}_{2}$ by TBL dots. The CL intensity increased proportionally with the concentration of added ${ }^{1} \mathrm{O}_{2}$, and the limit of detection (LOD) was calculated to be $1.26 \times 10^{-7} \mathrm{M}$ by $3 \mathrm{~S}_{0} / \mathrm{K}$ principle (Figure $\mathrm{S} 15$ ). Interestingly, the CL emission of TBL dots could last continuously for over one hour (Figure S16), and the CL intensity exhibited a great linear relationship with the concentration of TBL dots (Figure S17). The above results suggest that TBL dots can be employed as a ${ }^{1} \mathrm{O}_{2}$ indicator in analytical chemistry.

The cell viability and hemolysis test of TBL dots were performed before in vivo tissue imaging. As indicated in Figure S18A, TBL dots exhibited very low cytotoxicity over both 4T1 and NIH 3 T3 cells even when the concentration was as high as $200 \mu \mathrm{M}$. TBL dots also displayed great biocompatibility in hemolysis test, referenced by $0.2 \%$ Triton X-100 (Figure S18B). We 
next characterized the CL penetration depth of TBL dots by covering the reaction well with different slices of $\sim 3.0 \mathrm{~mm}$-thick pork ham (Figure 3A). Remarkably, our experiment manifested that the NIR CL emission of TBL dots could penetrate through 10 slices of pork ham, which was identical to a tissue thickness of over 30 $\mathrm{mm}$. As a comparison, the FL emission of TBL dots failed to penetrate through even one slice of pork ham. The tremendous advantage of CL emission over FL emission in tissue penetration could be attributed to the facts that CL emission does not require any excitation light and it can avoid the autofluorescence from tissue as well. The measured CL signals of TBL dots with different concentration of ${ }^{1} \mathrm{O}_{2}$ covered with one slice of pork ham was shown in Figure 3B. The signals through the tissue increased with the addition of ${ }^{1} \mathrm{O}_{2}$ concentration, suggesting they may reflect the concentration of ${ }^{1} \mathrm{O}_{2}$ qualitatively. In addition, the CL imaging of TBL dots was measured in comparison with that of luminol dots when both of them were covered by a slice of pork ham (Figure 3C). Although CL signals of TBL dots were 4.8 times lower than luminol dots in PBS solutions, the CL signals of TBL dots penetrating through the pork ham were 4.3 times higher than those of luminol dots (Figure S21), which verified the better performance of NIR TBL dots than blue luminol dots in deep-tissue penetration.

Subsequently, we evaluated the in vivo imaging of artificial ${ }^{1} \mathrm{O}_{2}$ by injecting a mixed solution of TBL dots $(2 \mathrm{mM}, 50 \mu \mathrm{L}), \mathrm{H}_{2} \mathrm{O}_{2}$ $(1 \mathrm{mM}, 50 \mu \mathrm{L})$, and $\mathrm{NaClO}(1 \mathrm{mM}, 50 \mu \mathrm{L})$ subcutaneously into the back of an anesthetized mouse (Figure 4). The CL emission decreased over time, which was similar to the in vitro kinetic curve (Figure S16). However, the signal-to-noise ratio of the CL signal still remained as high as 54 even after 60 minutes (Figure S22), which confirmed the possibility of using TBL dots for ${ }^{1} \mathrm{O}_{2}$ imaging in real physiological systems.

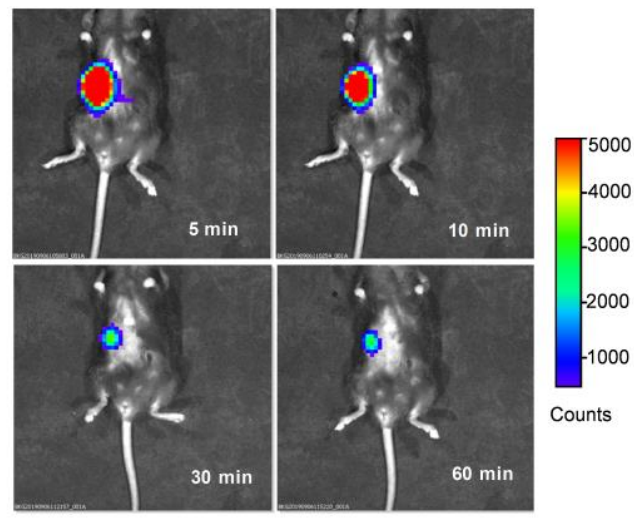

Figure 4. In vivo images of CL signals in hair-shaved mice (leftside body) after subcutaneous injection of TBL dots $(2 \mathrm{mM}, 50$ $\mu \mathrm{L})$ with $\mathrm{H}_{2} \mathrm{O}_{2}(1 \mathrm{mM}, 50 \mu \mathrm{L})$ and $\mathrm{NaClO}(1 \mathrm{mM}, 50 \mu \mathrm{L})$ for 5 min, $10 \mathrm{~min}, 30 \mathrm{~min}$ and $60 \mathrm{~min}$. Binning: 16; Exposure time: 1 min; f/Stop: 1 .

More prominently, the CL imaging of TBL dots in vivo can unambiguously differentiate tumor tissues from normal ones, with the amplification of $\mathrm{H}_{2} \mathrm{O}_{2}$ that is commonly used for clinical disinfection. ${ }^{26}$ As observed in Figure 5, when the mixture of TBL dots and $\mathrm{H}_{2} \mathrm{O}_{2}$ was injected into the tumor and normal areas respectively, the intensity of CL signals in the tumor site was significantly higher than in the normal tissue. In this situation, the added $\mathrm{H}_{2} \mathrm{O}_{2}$ could react with $\mathrm{HClO}$, the concentration of which was considerably higher in tumor microenvironment than in normal tissues, ${ }^{27}$ to generate large amount of ${ }^{1} \mathrm{O}_{2}$ and $\mathrm{HCl}$ in the tumor site, therefore facilitating the CL generation reaction by TBL. In addition, the generated $\mathrm{HCl}$ could further react with $\mathrm{H}_{2} \mathrm{O}_{2}$, catalyzed by myeloperoxidase that had a high expression in tumorassociated neutrophils, ${ }^{28-30}$ to produce more $\mathrm{HClO}$, which was also favorable to the CL process in the tumor sites. The selective illumination of tumor sites by CL emission of TBL dots revealed the great potential of TBL dots for CL-guided deep-tissue cancer diagnosis and surgery.
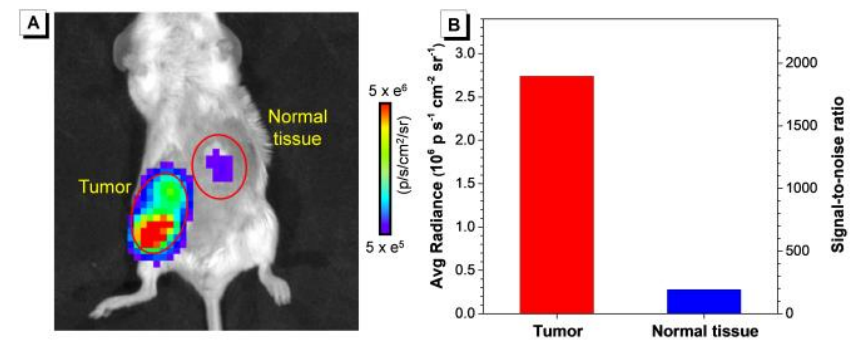

Figure 5. (A) In vivo CL images, (B) intensity and signal-to-noise ratio of tumor (left) and normal tissue (right) after injection of TBL dots $(2 \mathrm{mM}, 200 \mu \mathrm{L})$ with $\mathrm{H}_{2} \mathrm{O}_{2}(400 \mathrm{mM}, 50 \mu \mathrm{L})$. Binning:16; Exposure time: $2 \mathrm{~s}$; f/Stop: 1 .

In conclusion, we have designed and synthesized a new NIR CL emitter TBL by chemically conjugating luminol with benzothiadiazole and triphenylamine. The electron-withdrawing group benzothiadiazole can facilitate the CL process, and the AIE property of TBL ensures high NIR CL emission of TBL dots in aqueous solutions. In vitro and in vivo experiments have been conducted to evaluate the potential application of TBL dots for ${ }^{1} \mathrm{O}_{2}$ detection in real biological systems. The NIR CL emission could penetrate through $3 \mathrm{~cm}$-thick pork ham, showing great advantages over FL and blue CL emission. Furthermore, NIR CL emission of TBL dots can distinguish tumor tissues from normal ones successfully, which demonstrates the potential of this system for CLguided cancer diagnosis and surgery.

\section{ASSOCIATED CONTENT}

\section{Supporting Information}

The Supporting Information is available free of charge on the ACS Publications website.

Detailed methods, characterizations and supplementary figures (PDF)

\section{AUTHOR INFORMATION}

\section{Corresponding Authors}

*liangluo@ hust.edu.cn

*tangbenz@ust.hk

\section{ORCID}

Liang Luo: 0000-0001-9274-1866

Ben Zhong Tang: 0000-0002-0293-964X

\section{Author Contributions}

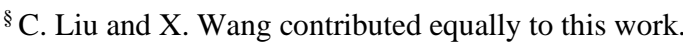

\section{Notes}

The authors declare no competing financial interests.

\section{ACKNOWLEDGMENT}

We are grateful for financial support from the National Science Foundation of China (21788102), the Research Grants Council of Hong Kong (N_HKUT609/19, C6009-17G and A-HKUST 605/16), the Innovation and Technology Commission (ITCCNERC14SC01 and ITCPD/17-9), Ming Wai Lau Centre for 
Reparative Medicine, Karolinska Institute (MWLC19Sc02), and the National Basic Research Plan of China (2018YFA0208903).

\section{REFERENCES}

(1) Wolfbeis, O. S. An Overview of Nanoparticles Commonly Used in Fluorescent Bioimaging. Chem. Soc. Rev. 2015, 44 (14), 4743-68.

(2) Chan, J.; Dodani, S. C.; Chang, C. J. Reaction-Based Small-Molecule Fluorescent Probes for Chemoselective Bioimaging. Nat. Chem. 2012, 4 (12), 973-84

(3) Yao, J.; Yang, M.; Duan, Y. Chemistry, Biology, and Medicine of Fluorescent Nanomaterials and Related Systems: New Insights into Biosensing, Bioimaging, Genomics, Diagnostics, and Therapy. Chem. Rev. 2014, 114 (12), 6130-78.

(4) Kobayashi, H.; Ogawa, M.; Alford, R.; Choyke, P. L.; Urano, Y. New Strategies for Fluorescent Probe Design in Medical Diagnostic Imaging. Chem. Rev. 2010, 110 (5), 2620-2640.

(5) Weissleder, R.; Ntziachristos, V., Shedding Light onto Live Molecular Targets. Nat. Med. 2003, 9 (1), 123-128.

(6) Baumes, J. M.; Gassensmith, J. J.; Giblin, J.; Lee, J. J.; White, A. G.; Culligan, W. J.; Leevy, W. M.; Kuno, M.; Smith, B. D. Storable, Thermally Activated, Near-Infrared Chemiluminescent Dyes and Dye-Stained Microparticles for Optical Imaging. Nat. Chem. 2010, 2 (12), 1025-30.

(7) Frangioni, J. V. The Problem is Background, not Signal. Mol. Imaging 2009, 8 (6), 303-4.

(8) Zomer, G. Chapter 2. The Nature of Chemiluminescent Reactions. In Chemiluminescence and Bioluminescence, 2010, pp 51-90.

(9) Giussani, A.; Farahani, P.; Martinez-Munoz, D.; Lundberg, M.; Lindh, R.; Roca-Sanjuan, D. Molecular Basis of the Chemiluminescence Mechanism of Luminol. Chem. Eur. J. 2019, 25 (20), 5202-5213.

(10) Green, O.; Gnaim, S.; Blau, R.; Eldar-Boock, A.; Satchi-Fainaro, R.; Shabat, D. Near-Infrared Dioxetane Luminophores with Direct Chemiluminescence Emission Mode. J. Am. Chem. Soc. 2017, 139 (37), 13243-8.

(11) Yamaguchi, S.; Kishikawa, N.; Ohyama, K.; Ohba, Y.; Kohno, M.; Masuda, T.; Takadate, A.; Nakashima, K.; Kuroda, N. Evaluation of Chemiluminescence Reagents for Selective Detection of Reactive Oxygen Species. Anal. Chim. Acta. 2010, 665 (1), 74-8.

(12) Zhang, X.; Xu, Y.; Giordano, F.; Schreier, M.; Pellet, N.; Hu, Y.; Yi, C.; Robertson, N.; Hua, J.; Zakeeruddin, S. M.; Tian, H.; Gratzel, M. Molecular Engineering of Potent Sensitizers for Very Efficient Light Harvesting in Thin-Film Solid-State Dye-Sensitized Solar Cells. J. Am. Chem. Soc. 2016, 138 (34), 10742-5.

(13) Justin Thomas, K. R.; Lin, J. T.; Velusamy, M.; Tao, Y. T. Chuen, C. H., Color Tuning in Benzo[1,2,5]thiadiazole-Based Small Molecules by Amino Conjugation/Deconjugation: Bright Red-Light-Emitting Diodes. Adv. Funct. Mater. 2004, 14 (1), 83-90.

(14) Lee, W. W. H.; Zhao, Z.; Cai, Y.; Xu, Z.; Yu, Y.; Xiong, Y.; Kwok, R. T. K.; Chen, Y.; Leung, N. L. C.; Ma, D.; Lam, J. W. Y.; Qin, A.; Tang, B. Z. Facile Access to Deep Red/Near-Infrared Emissive AIEgens for Efficient Non-Doped OLEDs. Chem. Sci. 2018, 9 (28), 6118-6125.

(15) Han, J.; Jose, J.; Mei, E.; Burgess, K., Chemiluminescent EnergyTransfer Cassettes Based on Fluorescein and Nile Red. Angew. Chem. Int. Ed. Engl. 2007, 46 (10), 1684-7.

(16) Mei, J.; Leung, N. L.; Kwok, R. T.; Lam, J. W.; Tang, B. Z. Aggregation-Induced Emission: Together We Shine, United We Soar! Chem. Rev. 2015, 115 (21), 11718-940.

(17) Qian, J.; Tang, B. Z. AIE Luminogens for Bioimaging and Theranostics: From Organelles to Animals. Chem 2017, 3 (1), 56-91.

(18) Liu, S.; Zhang, H.; Li, Y.; Liu, J.; Du, L.; Chen, M.; Kwok, R. T. K.; Lam, J. W. Y.; Phillips, D. L.; Tang, B. Z. Strategies to Enhance the Photosensitization: Polymerization and the Donor-Acceptor Even-Odd Effect. Angew. Chem. Int. Ed. Engl. 2018, 57 (46), 15189-15193.

(19) Xu, S.; Duan, Y.; Liu, B. Precise Molecular Design for HighPerformance Luminogens with Aggregation-Induced Emission. Adv. Mater. 2020, 32 (1), e1903530.

(20) Kim, T. G.; Castro, J. C.; Loudet, A.; Jiao, J. G. S.; Hochstrasser, R. M.; Burgess, K.; Topp, M. R. Correlations of Structure and Rates of Energy Transfer for Through-Bond Energy-Transfer Cassettes. J. Phys. Chem. A 2006, 110 (1), 20-27.

(21) Zhang, S.; Cui, H.; Gu, M.; Zhao, N.; Cheng, M.; Lv, J. Real-Time Mapping of Ultratrace Singlet Oxygen in Rat during Acute and Chronic Inflammations via a Chemiluminescent Nanosensor. Small 2019, 15 (16), e1804662.
(22) Mao, D.; Wu, W.; Ji, S.; Chen, C.; Hu, F.; Kong, D.; Ding, D.; Liu, B. Chemiluminescence-Guided Cancer Therapy Using a Chemiexcited Photosensitizer. Chem 2017, 3 (6), 991-1007.

(23) Ni, X.; Zhang, X.; Duan, X.; Zheng, H. L.; Xue, X. S.; Ding, D. Near-Infrared Afterglow Luminescent Aggregation-Induced Emission Dots with Ultrahigh Tumor-to-Liver Signal Ratio for Promoted ImageGuided Cancer Surgery. Nano. Lett. 2019, 19 (1), 318-330.

(24) Adam, W.; Kazakov, D. V.; Kazakov, V. P. Singlet-Oxygen Chemiluminescence in Peroxide Reactions. Chem. Rev. 2005, 105 (9), 33713387.

(25) Kanofsky, J. R. Singlet Oxygen Production by ChloroperoxidaseHydrogen Peroxide-Halide Systems. J. Biol. Chem. 1984, 259 (9), 5596600.

(26) Rutala, W. A.; Weber, D. J. Disinfection and Sterilization in Health Care Facilities: What Clinicians Need to Know. Clin. Infect. Dis. 2004, 39 (5), 702-709.

(27) Zhu, H.; Fan, J.; Wang, J.; Mu, H.; Peng, X. An "Enhanced PET"Based Fluorescent Probe with Ultrasensitivity for Imaging Basal and Elesclomol-Induced HClO in Cancer Cells. J. Am. Chem. Soc. 2014, 136 (37), 12820-3.

(28) Kettle, A. J.; Winterbourn, C. C. Myeloperoxidase: A Key Regulator of Neutrophil Oxidant Production. Redox Rep. 1997, 3 (1), 3-15.

(29) Haegens, A.; Vernooy, J. H.; Heeringa, P.; Mossman, B. T.; Wouters, E. F. Myeloperoxidase Modulates Lung Epithelial Responses to Pro-Inflammatory Agents. Eur. Respir. J. 2008, 31 (2), 252-60.

(30) Rymaszewski, A. L.; Tate, E.; Yimbesalu, J. P.; Gelman, A. E.; Jarzembowski, J. A.; Zhang, H.; Pritchard, K. A., Jr.; Vikis, H. G. The Role of Neutrophil Myeloperoxidase in Models of Lung Tumor Development. Cancers (Basel) 2014, 6 (2), 1111-27. 
Table of Contents (TOC)

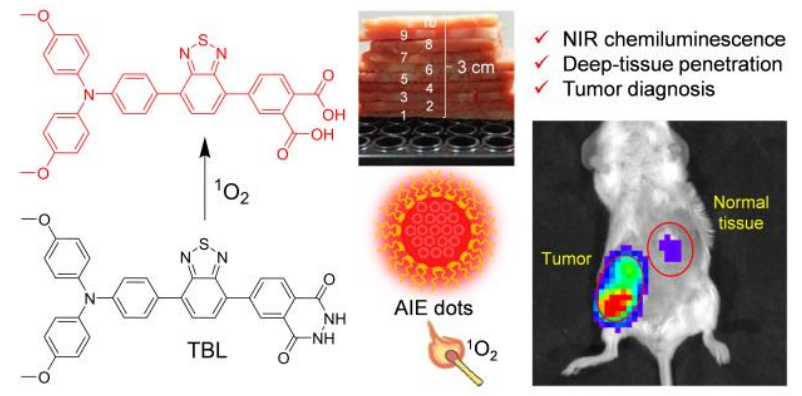

